\title{
Social attitudes differentially modulate imitation in adolescents and adults
}

\author{
Jennifer Cook $\cdot$ Geoffrey Bird
}

Received: 5 September 2010/Accepted: 29 January 2011/Published online: 19 February 2011

(C) The Author(s) 2011. This article is published with open access at Springerlink.com

\begin{abstract}
Previous studies have demonstrated a bidirectional relationship between social attitudes and imitation in adults: pro-social attitudes promote imitation, and imitation further increases positive social attitudes. Social attitudes and the social brain are developing throughout the adolescent years. Thus, the aim of this study was to test whether pro-social attitudes promote imitation in an Adolescent Group to the same extent as in an Adult Group. Participants were primed with pro-social or non-social words in a Scrambled Sentence Priming task. They then completed an Imitation task wherein participants were required to perform a lift action with either the index or middle finger, whilst observing either a compatible action (e.g. index finger response and observed index finger lift) or an incompatible action (e.g. index finger response and observed middle finger lift). In an Effector Priming control condition, observed fingers remained stationary but a semitransparent green mask was added to either the compatible or incompatible finger. The magnitude of the Imitation Effect and Effector Priming Effect was calculated by subtracting reaction times on compatible trials from those on incompatible trials. In the Adult Group, social priming specifically modulated the Imitation Effect: pro-social priming produced a larger Imitation Effect but did not modulate the Effector Priming Effect. In adolescents, however, no effect of social priming was seen on either the Imitation or Effector Priming measures. We consider
\end{abstract}

J. Cook · G. Bird $(\bowtie)$

Institute of Cognitive Neuroscience, University College London,

17 Queen Square, London WC1N 3AR, UK

e-mail: g.bird@bbk.ac.uk

G. Bird

Department of Psychological Sciences,

Birkbeck College, Malet Street, London WC1E 7HX, UK possible explanations for these results including the immature development of social brain regions and reduced experience of the relationship between social attitudes and imitation in adolescence.

Keywords Imitation - Adolescence · Priming · Chameleon effect $\cdot$ Social

\section{Introduction}

Joint action has been defined as a social interaction whereby two or more individuals coordinate their actions in space and time to bring about a change in the environment (Sebanz et al. 2006). One example of this is the 'Joint Simon' task in which participants see one of two cues (e.g. red and green squares) appear on either the left or right of a screen and are told that they should respond to a specific cue (e.g. red square) and that another participant will respond to the other cue. The knowledge that another participant is responding to the redundant cue results in a Simon effect that persists even if the participant cannot see the actions of the other individual. That is, despite not seeing the action of the other participant, there is a significant effect of spatial compatibility on response time which is normally only seen when the participant must make differential responses to both cues (Sebanz et al. 2003). Another example of joint action is imitation. Imitation is a form of joint action in which multiple actors may coordinate their actions in space and time on the basis of the actions they observe. By studying mechanisms of imitation, we can also make progress in understanding the mechanisms underlying other forms of joint action.

There is increasing evidence that unconscious imitation promotes positive social attitudes (Chartrand and Bargh 
1999; Lakin and Chartrand 2003). It has been demonstrated that when people are not aware that their behaviour is being copied, being mimicked increases rapport (Chartrand and Bargh 1999), feelings of closeness (Kühn et al. 2010), altruistic behaviour (van Baaren et al. 2004) and trust (Bailenson and Yee 2005). In a study where half the participants were mimicked by a confederate and half were not, participants who were mimicked reported liking the confederate more than those who were not (Chartrand and Bargh 1999). In another study, waitresses who were instructed to mimic their customers received bigger tips than those who were instructed not to mimic (van Baaren et al. 2003a). Thus, a range of studies suggests that unconscious mimicry is linked to increased positive social attitudes.

A number of studies suggest that the converse relationship-that positive social attitudes increase unconscious mimicry - is also true. Early research suggested that among romantic couples, there is a correlation between the amount of rapport they feel with each other and the amount of imitation that takes place during their interactions (Bernieri 1988; Lafrance 1979, 1982; Lafrance and Broadbent 1976). Van Baaren et al. varied self-construal orientation and found that participants with either a temporarily induced, or chronic, dominant interdependent selfconstrual were more likely to match the behaviours of a confederate than those with an independent self-construal (van Baaren et al. 2003b). Recently Leighton et al. (2010) demonstrated that unconscious priming of positive social attitudes increases imitative behaviour in adults. In the study by Leighton et al., each participant completed two tasks: first, a social attitude priming task and then an automatic imitation task. For the priming task, participants formed sentences from sets of words that included prosocial, neutral or anti-social terms (three groups). In each trial of the automatic imitation task, participants were required to perform a pre-specified hand movement (open or close) as soon as they saw a hand on a computer screen begin to move. The hand movement stimulus was either the same as the pre-specified response (compatible trials, e.g. open stimulus and open response) or the hand movement stimulus was the opposite of the pre-specified response (incompatible trials, e.g. close stimulus and open response). Although participants are not required to imitate, imitative responses (compatible trials) are consistently faster than non-imitative responses (incompatible trials; Brass et al. 2000; Heyes et al. 2005). The magnitude of this Imitation Effect was calculated as the reaction time difference between incompatible and compatible trials. Leighton et al. found that participants primed with pro-social words showed a larger Imitation Effect than those primed with anti-social words, and the neutral group showed an intermediate Imitation Effect.
Although the psychological and neural mechanisms by which social attitudes modulate imitation are currently unknown, a number of studies suggest that the neural mechanisms that underlie the representation of others are involved in 'top-down' control of automatic imitative responses (Brass et al. 2003, 2005; Spengler et al. 2010a, b). In a study using functional Magnetic Resonance Imaging (fMRI), Brass et al. (2005) demonstrated that inhibition of imitation, in contrast to inhibition of nonimitative automatic responses (Stroop Task), activated both the medial prefrontal cortex (MPFC) and temporo-parietal junction (TPJ). The MPFC and TPJ are part of the "social brain' (Brothers 1990; Frith 2007; Frith and Frith 2010), a network of brain areas that is active when we process social information, in particular when we represent the mental states (intentions, desires, beliefs) of others ("theory of mind'). In addition to recruiting common neural areas, two recent studies suggest that the control of imitation and the representation of others are functionally associated. Spengler et al. (2010b) showed that the degree to which participants were able to inhibit imitative responses was correlated with performance on a behavioural theory of mind task and also with activity in the MPFC and TPJ when participants performed a theory of mind task whilst being scanned. Furthermore, patients with lesions to the MPFC and TPJ show impaired performance on theory of mind tasks, and the extent of their impairment correlates with their degree of impairment on an imitation inhibition task (Brass et al. 2003). Therefore, these studies suggest that control of imitation and the representation of others share a component process, and that parts of the social brain may be responsible for mediating imitation as a result of social priming.

Adolescence is an important period in the development of social cognition and the social brain (for review see Blakemore 2008, 2010). This period can be characterized by changes in self-awareness (Sebastian et al. 2008; Harter 1990), in social behaviour (Brown 2004), and in responses to social situations (Sebastian et al. 2010; Hillebrandt et al. in press). For instance, Sebastian et al. (2010) showed that self-reported affective responses to social exclusion are significantly greater for adolescents compared to adults.

In addition to changes in social attitudes and behaviours, the adolescent years are also a time when many structural and functional changes are taking place in the brain. Both cellular (Huttenlocher 1979) and MRI (Giedd et al. 1999; Sowell et al. 1999; Paus 2005) studies suggest that some brain regions, including parts of the social brain, are still anatomically developing well into the adolescent years. For example, a recent developmental MRI study that measured cortical thickness in a sample of 375 participants aged 4-21 found that MPFC is one of the latest regions to mature, with a peak in cortical thickness at around age 14 years 
(Shaw et al. 2008). Recent neuroimaging studies also suggest functional maturation of the social brain that continues throughout adolescence. A number of studies have reported increased activation of the MPFC in adolescents relative to adults when representing the mental states (Blakemore et al. 2007; Wang et al. 2006) or the emotions (Burnett et al. 2009) of others. These studies suggest that parts of the social brain, which may be responsible for mediating imitation as a result of social priming, are still maturing throughout the adolescent years. Therefore, this experiment investigates development in adolescence of the modulation of automatic imitation by social attitudes.

In the current experiment, we used a modified version of the Leighton paradigm. As in the study by Leighton et al., each participant completed two tasks: a social attitude priming task followed by an imitation task. In the priming task, participants formed sentences from sets of words that included either pro-social terms or non-social terms (two groups). In each trial of the imitation task, which was a modified version of the two alternative choice reaction time task used in Brass et al. (2000), participants were required to perform a lift action with either the index or middle finger as soon as they saw a cue appear on screen. Concurrently, participants observed a video of a finger lift. In $50 \%$ of trials, the observed effector was compatible with the effector that the participant was required to use (e.g. index finger response and observed index finger lift), and in $50 \%$ of trials, the observed effector was incompatible (e.g. index finger response and observed middle finger lift). We hypothesized that the effect of social priming on imitation would differ as a function of age group.

In the original, naturalistic, experiments demonstrating a link between pro-social attitudes and imitation (Lakin and Chartrand 2003), it was unclear whether pro-social priming increased imitation specifically. Imitation was measured by scoring matching behaviour in naturalistic social interactions. In the majority of these studies, a participant interacted with a confederate who either shook their foot or rubbed their face with above average frequency. Judges measured the frequency of each action for each participant, yielding an 'imitation score'. For example, imitation involved foot shaking in the presence of a foot shaking confederate but not in the presence of a face-rubbing confederate. However, this paradigm does not allow one to answer the question of whether social attitudes modulate imitation of another's specific movements, or whether they just copy the degree to which the effector is moved. Observing a foot shaking model may result in increased performance of a variety of foot movement, which may indirectly increase the proportion of foot shaking. Nonspecific effects of this kind are known in the motor control literature as 'effector priming' (Bach and Tipper 2007; Berger and Hadley 1975; Gillmeister et al. 2008).
In contrast, the effect reported by Leighton et al. was specific to the movement type required. Pro-socially primed adults were faster to conduct a hand-open response when observing a hand-open response rather than being faster to open their hand when observing any hand movement. Leighton et al. therefore argue that modulation of imitation by social attitudes does not occur indirectly via effector priming. To test this hypothesis, we added a control condition to our imitation task wherein no finger movement was present, instead a semi-transparent green mask was superimposed over either the compatible or incompatible finger. The green mask draws attention to the effector but does not afford imitation since no movement is present. It has previously been demonstrated that drawing attention to an effector, even if the effector is static, produces speeding of responses made with that effector. For example, Bach and Tipper (2007) showed that presenting participants with photographs of individuals with a coloured dot on either their hands or their feet speeded responses made by participants using the same effector. We hypothesized that adult participants primed with pro-social words would show a greater Imitation Effect than those primed with non-social words in the Imitation task but that performance on the Effector Priming Control task would be unaffected by social priming.

\section{Methods}

\section{Participants}

The study included 90 participants divided into four groups: Non-social Adolescent Group $(N=17$, mean age $=13.71$, Standard Error $(\mathrm{SEM})=0.19)$; Pro-social Adolescent Group $(N=17$, mean age $=13.82, \mathrm{SEM}=$ 0.18); Non-social Adult Group $(N=28$, mean age $=$ 28.07, $\mathrm{SEM}=2.58)$; Pro-social Adult Group $(N=28$, mean $=25.79$, SEM $=2.00)$. For each age group, the Non- and Pro-social groups were matched in terms of age (Adolescent Groups: $t(32)=-0.46, P=0.65$ (2-tailed); Adult Groups: $t(54)=0.70, P=0.49$ (2-tailed)). Adults were recruited from the University College London Psychology volunteer database, and adolescents were recruited from two local schools. Within these age groups, the participants were randomly allocated to the Pro-social or Nonsocial Group. All participants were naïve with respect to the purpose of the experiment. Informed consent was obtained from all adults and from the primary caregiver of each adolescent participant. The study was approved by the local ethics committee and performed in accordance with the ethical standards laid down in the 1964 Declaration of Helsinki. 


\section{Procedure}

\section{Priming task}

A modified version of the Scrambled Sentence Priming task used in Leighton et al. (2010) was employed. There were 36 trials in total. Each trial showed five words arranged vertically down the centre of the screen. The task was to pick 4 of the 5 words to make a grammatically correct sentence. Words were presented in Arial, font size 24. The colour of the words (red, green, black or blue) varied randomly over trials in order to suggest to the participant that the aim of the task was to investigate the effect of colour on word processing and hence distract from the priming nature of the task.

Two versions of the priming task were used: one to prime a pro-social attitude, one to prime a non-social attitude. For both versions, 24 of the 36 trials contained a priming word, that is, they contained an adjective or verb semantically related to the target attitude (pro-social or non-social). Twelve of the sentences were neutral (e.g. "the car was small"). This corresponds to a 2/3 saturation level, which has previously been shown to be effective for priming target attitudes without provoking conscious awareness of this target attitude (R. Van Baaren, personal communication). For the pro-social priming version, the 24 priming words were: friend, talkative, sociable, married, outgoing, crowded, cooperative, agreeable, family, friendly, group, others, team, chatty, gathering, together, unity, sharing, joined, interactions, society, meeting, community, popularity (e.g., "he is very talkative"). For the non-social priming version, the 24 priming words were rebel, selfish, alone, single, independent, withdrawn, secluded, uncooperative, disagreeable, independence, private, himself, individual, think, solitary, solo, detached, lone, separate, one, isolated, personal, self, unpopular (e.g. "she is often withdrawn"). Priming task duration was approximately $15 \mathrm{~min}$.

\section{Automatic Imitation and Effector Priming Control Tasks}

In order to obtain a reaction time (RT) measure of automatic imitation in a classroom/laboratory setting, we used a modified version of the two alternative choice reaction time task used in Brass et al. (2000). Short video sequences of a human hand were presented on the computer screen. The video sequences subtended approximately $6^{\circ}$ visual angle vertically and $9^{\circ}$ visual angle horizontally and consisted of five full-colour frames presented on a black background. The hand was presented in the vertical orientation (with the fingers pointing to the right), whereas the participant rested their hand in a horizontal orientation (fingers point to the computer screen) on the computer keypad; hence, response movements were orthogonal to stimulus postures. This feature of the design allows automatic imitation to be isolated from spatial compatibility.

The Imitation and Effector Priming Control tasks comprised a $2 \times 2$ factorial design with the factors Compatibility (between participant's finger and on screen finger: Compatible, Incompatible) and Experimental Task (Imitation Task, Effector Priming Control Task). For 50\% of the compatible trials, the finger lifted (or changed to a green colour in the Effector Priming Control Task) was the index finger, and for $50 \%$ of the incompatible trials, the manipulated finger was the middle finger. There were 30 trials per trial type, 120 trials in total. Trials from the Imitation and Effector Priming Control Tasks were pseudo-randomized such that one trial type never occurred more than twice in a row. The duration of the Imitation plus Effector Priming Control Task was approximately $15 \mathrm{~min}$.

On each trial, the participant was required to place the index finger of their right hand on the letter ' $\mathrm{V}$ ' on the computer keyboard and the middle finger of their right hand on the letter ' $\mathrm{B}$ ' on the keyboard. Participants were told to lift the finger up as quickly as possible and then replace it on the same key. They should lift their index finger if a number 1 appeared on the screen and lift their middle finger if a number 2 appeared on the screen. The imperative stimulus (the 1 or 2 ) appeared between the index and middle fingers and marked the onset of the response period. After the appearance of the imperative stimulus, the stimulus video was presented for 3,000 ms. The nature of the stimulus video depended on whether the trial was of the Imitation Task, or the Effector Priming Control Task.

\section{Imitation task}

In $50 \%$ of Imitation Task trials, the video sequence depicted an action that was compatible with the participant's required movement (e.g. the participant was required to lift the index finger, and the video showed an index finger lift); on $50 \%$ of trials, the video showed an incompatible action (e.g. the participant was required to lift the index finger and the video showed a middle finger lift). The RT difference between incompatible and compatible trials was used as an index of automatic imitation (Imitation Effect).

\section{Effector priming control task}

Stimulus videos used in the Effector Priming Control Task were not of an action. Rather, one of the fingers acquired a semi-transparent green mask (Fig. 1b). On 50\% of trials, the finger that changed colour was compatible with the instructed response (e.g. an index finger response was 
required, and the colour of the index finger changed to green), and on $50 \%$ of trials, the finger that changed colour was incompatible with the required response (e.g. an index finger response was required, and the middle finger changed colour). The RT difference between incompatible and compatible trials generated an index of effector priming (Effector Priming Effect).

\section{Stimuli}

In both the Effector Priming Control Task and Imitation Task, stimuli were presented on a computer screen at a distance of approximately $57 \mathrm{~cm}$.

Stimulus videos in the Imitation task were comprised of five frames (Fig. 1a). The first was a photograph of a hand at rest against a blue worktop. This frame was displayed for a variable interval with range $800-2,400 \mathrm{~ms}$ and acted as a warning signal. The second frame was a photograph of the index or middle finger $1 / 3$ rd of the way through a lifting action and a number 1 or 2 . The third was a photograph of the same finger $2 / 3$ of the way through a lifting action. These frames were displayed for $34 \mathrm{~ms}$. The fourth frame was a photograph of the finger in the fully lifted position, which was displayed for $500 \mathrm{~ms}$. These display durations ensured the appearance of a short video clip of a finger being lifted with the concurrent appearance of a number. The fifth frame was a blank screen. This frame remained on screen until the duration of the trial had reached $3,000 \mathrm{~ms}$ and until the participant had returned both fingers to the letters $\mathrm{V}$ and $\mathrm{B}$ on the keyboard.

Stimulus videos in the Effector Priming Control task were comprised of three frames (Fig. 1b). The first was a photograph of a hand at rest, displayed for a variable interval with range 800-2,400 ms. For the second frame, the finger in the photograph was coloured green, and a number 1 or 2 was added to the image. This frame was displayed for $568 \mathrm{~ms}$. The third frame was a blank screen the same blue as the background in frames 1 and 2. This frame remained on screen until the duration of the trial had reached 3,000 $\mathrm{ms}$ and until the participant had returned both fingers to the response keys; hence, both Imitation and Effector Priming Control Trial types were of the same duration.

\section{Testing procedure}

Participants were tested in groups of approximately 10-20 individuals under exam conditions; adolescents were tested in their school computer cluster room, and adults were tested in a computer cluster room at UCL. Participants
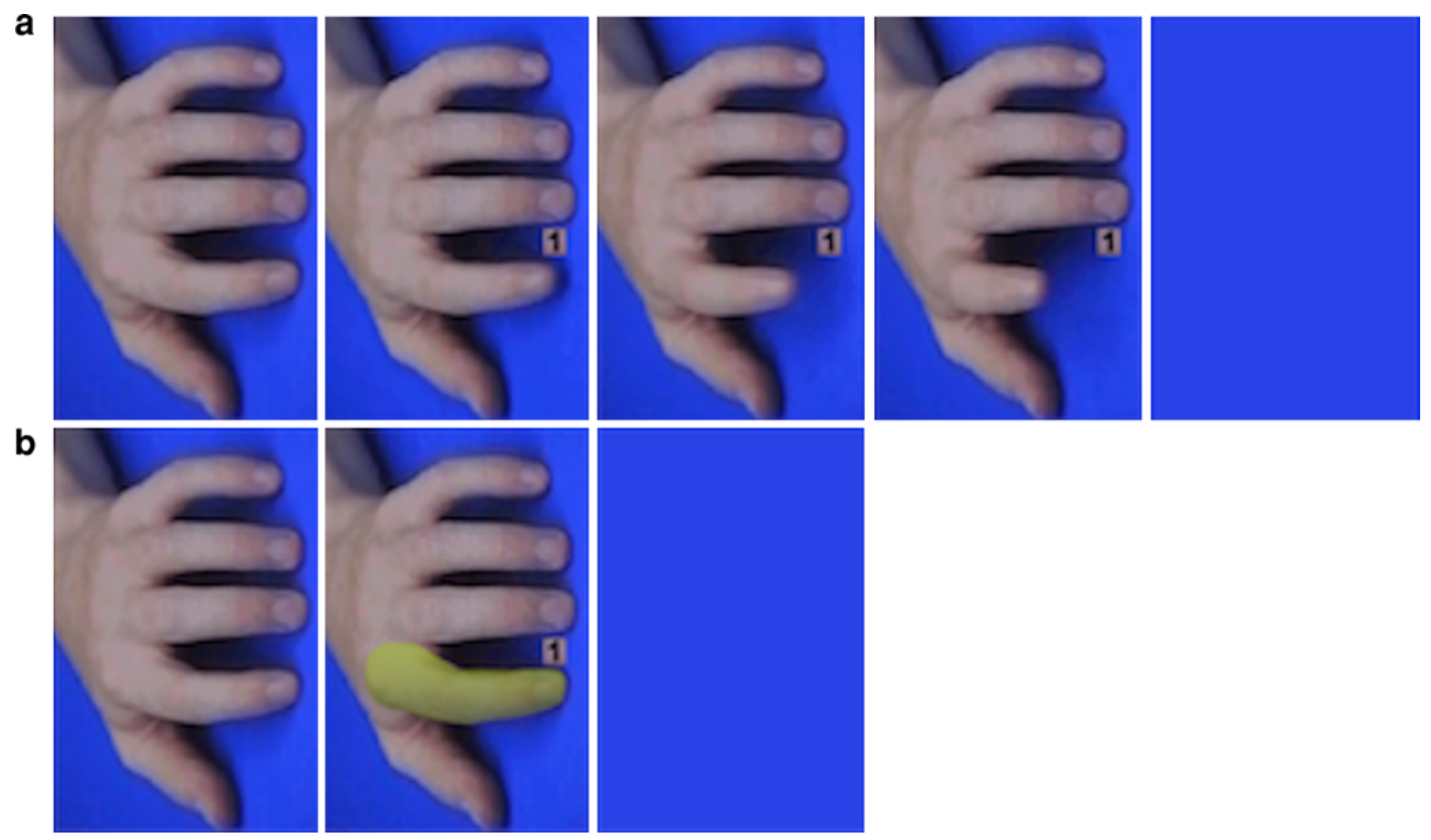

Fig. 1 a The five frames of an Imitation Task trial. The frames shown here constitute a compatible video where the participant must lift their index finger whilst watching an index finger lift. Video duration was $3,000 \mathrm{~ms}$. b The three frames of an Effector Priming
Control trial. The frames shown here constitute a compatible Effector Priming trial where the participant must lift their index finger, whilst viewing an index finger highlighted by a green mask. Video duration was $3,000 \mathrm{~ms}$ 
were informed that they would be asked to take part in two separate experiments, one on the effect of colour on word processing and the other on responses to numbers. Prior to testing all participants were read standardized instructions and completed 5 example trials of the Scrambled Sentence Priming task. Participants were shown an example of each trial type from the Imitation and Effector Priming Control Tasks. All participants were also presented with a written version of the instructions. For both the pro- and non-social priming tasks, participants were told that they would see five words on the screen and that they should use four of the five words to make a grammatically correct sentence. The subjects were instructed to select the words by clicking on each one with the computer mouse. They were told that once they had clicked on the fourth and final word in their created sentence, a new screen would appear with five different words. For the Imitation and Effector Priming Control tasks, participants were told to place the index finger of their right hand on the letter ' $\mathrm{V}$ ' on the computer keyboard and the middle finger of their right hand on the letter 'B' on the keyboard. Participants were told to lift their index finger if a number 1 appeared on the screen and lift their middle finger if a number 2 appeared on the screen. Before the Imitation and Effector Priming Control tasks commenced, participants conducted a training session. The training programme required participants to make 5 correct consecutive responses in order for them to continue onto the experimental version of the task. The probability of making 5 correct consecutive responses by chance is $<0.05$; therefore, the training task ensured that participants could perform the task before data collection began.

Following the Imitation and Effector Priming Control tasks, participants were asked to complete a debriefing questionnaire designed to ascertain whether participants had guessed the purpose of the experiment (see Leighton et al. 2010). This questionnaire included the following questions: "What do you think the purpose of this experiment was? What do you think this experiment was trying to study? Did you think that any of the tasks were related in any way? If yes, in what way? Did anything you did on one task affect anything you did in another task? If yes, then how did it affect you? When you were arranging the words, did you notice anything unusual about the words? Did you notice a pattern or theme to the words? Did you have a particular goal or strategy when arranging the words?"

Data analysis

\section{Priming task}

For all participants, all of the sentences produced in the Priming task were scored. A grammatically incorrect sentence yielded an error score of 1. Error scores for all 32 sentences were then summed to give an error score across all sentences.

\section{Imitation and effector priming control tasks}

For the Imitation and Effector Priming Control tasks, errortrials in which the participant lifted the incorrect finger were removed from the analysis. RTs were filtered such that those less than $150 \mathrm{~ms}$ were excluded under the assumption that they were expectancy errors and those longer that 2,000 ms were excluded under the assumption that they reflected a lapse in attention.

\section{Results}

All results are reported using an $\alpha$ of $P<0.05$. Two-tailed tests are used except where a directional prediction has been made on the basis of pre-existing literature. When a one-tailed test has been used, this is indicated following a statistically significant result.

Examination of the debriefing questionnaire data indicated that no participant correctly guessed the purpose of either the Priming or Imitation and Effector Priming Control tasks. Furthermore, no participant correctly identified a link between the studies or a theme among the words presented in the Priming task. Therefore, we can conclude that no participant was aware of the type of priming they had received or that the purpose of the study was to examine imitation and its relationship with social attitudes.

\section{Priming task}

In the Priming task, no group made more than 2 errors on average (Adolescent Non-social mean $=1.41$; Adolescent Pro-social mean $=1.59$; Adult Non-social mean $=1.39$; Adult Pro-social mean $=0.93$ ). To test whether either Age or Prime Group significantly affected the number of errors, we employed a $2 \times 2$ ANOVA with between-subjects factors Age Group and Prime Group. There was no significant interaction between Age and Prime Group $(F(1,86)=0.63, P=0.43)$ and no main effect of Age Group $(F(1,86)=0.71, \quad P=0.40) \quad$ or Prime Group $(F(1,86)=0.13, P=0.72)$. To be sure that no one group produced more errors for the sentences which contained prime words, we created a 'priming sentence error score' by summing errors for the 24 sentences which contained a priming word. No group made more than 2 errors on average (Adolescent Non-social mean $=1.12$; Adolescent Pro-social mean $=1.06$; Adult Non-social mean $=1.07$; Adult Pro-social mean $=0.68$ ). To test whether Age or 
Prime Group had a significant effect on 'priming sentence error score', we employed a $2 \times 2$ ANOVA with betweensubjects factors Age Group and Prime Group. There was no significant interaction between Age and Prime Group $(F(1,86)=0.25, P=0.62)$ and no main effect of Age Group $(F(1,86)=0.42, P=0.52)$ or Prime Group $(F(1,86)=$ $0.47, P=0.50)$. Hence, all groups exhibited equivalent performance on the Priming task.

Imitation and effector priming tasks

\section{Error analysis}

Error rates (defined as incorrect responses and reaction times less than $150 \mathrm{~ms}$, and greater than 2,000 ms) were low in both the Imitation Task (7.9\% of trials) and the Effector Priming Task (6.5\% of trials). For the Imitation and Effector Priming tasks, errors on Incompatible trials are of theoretical relevance for the current study as they indicate a failure to inhibit responding on the basis of automatic imitation and effector priming, respectively. Therefore, errors on Incompatible trials were entered into a $2 \times 2$ ANOVA with factors of Age Group (Adult, Adolescence) and Prime Group (Pro-social, Non-social). For the Imitation Task, this ANOVA revealed a significant main effect of Age Group $(F(1,83)=10.0, P=0.002)$; however, the main effect of Prime Group and the interaction between Age and Prime Group were not significant (both $F(1,83)<1)$. The main effect of Age reflects an increased tendency to imitate in the Adolescent Group (Adolescent Group mean error rate $14.0 \%$ of Incompatible Trials, Adult error rate $7.3 \%$ of Incompatible trials) that was not affected by the Prime Group factor. The same ANOVA performed on the Effector Priming Task revealed a marginally significant effect of Age Group $F(1,83)=$ $3.9, P=0.051)$ but the main effect of Prime Group and the Prime $x$ Age Group interaction was not significant (both $F(1,83)<1)$. The main effect of Age reflects an increased tendency to respond on the basis of effector compatibility in the Adolescent Group (Adolescent Group mean error rate $9.8 \%$ of Incompatible Trials, Adult error rate $5.1 \%$ of
Incompatible trials) that was not affected by the Prime Group factor.

\section{RT analysis}

The magnitude of the Imitation Effect was calculated, for each participant, by subtracting RT on compatible trials from RT on incompatible Imitation task trials. Similarly, an index of Effector Priming Effect was calculated, for each participant, by subtracting RT in compatible trials from RT in incompatible trials from the Effector Priming Control Task. Table 1 shows the Imitation and Effector Priming Effects for each Prime Group in each Age Group.

Differential effects of pro-social/non-social priming on imitation in adolescents compared to adults

Paired samples $t$ tests on compatible and incompatible RTs for Imitation trials demonstrated that each of the four groups exhibited a significant Imitation Effect (Adolescent Nonsocial Group incompatible trials mean RT $($ SEM $)=547.87$ (23.50), compatible trials mean RT $(\mathrm{SEM})=497.26$ (18.05), $t(16)=4.22, P<0.001,1$-tailed; Adolescent Prosocial Group incomp mean RT $(\mathrm{SEM})=516.53$ (21.49), comp mean RT $(\mathrm{SEM})=478.55(17.01), t(16)=4.12$, $P<0.001$, 1-tailed; Adult Non-social Group incomp mean $\mathrm{RT}(\mathrm{SEM})=508.89(14.35)$, comp mean RT $(\mathrm{SEM})=$ 470.91 (12.90), $t(27)=5.28, P<0.001,1$-tailed; Adult Pro-social Group incomp mean RT $($ SEM $)=573.29$ (29.76), comp mean RT $(\mathrm{SEM})=502.18$ (22.13), $t(27)=$ $6.03, P<0.001$, 1-tailed). That is, for each group, responses were faster when observing a lift of the same finger than when observing a lift of a different finger. To examine whether social priming differentially affects adolescents and adults, we conducted a $2 \times 2$ ANOVA with between-subjects factors Age Group (Adolescent, Adult) and Prime Group (Pro-social, Non-social). The ANOVA revealed a significant interaction between Age and Prime Group $(F(1,86)=4.61, P=0.02,1$-tailed $)$. There was no main effect of Age Group $(F(1,86)=0.93, P=0.34$ or Prime Group $(F(1,86)=0.93, P=0.34)$. Post hoc independent

Table 1 Imitation and Effector Priming Effects for each Prime Group for each Age Group

\begin{tabular}{llllll}
\hline & Adolescent & & & \multicolumn{1}{c}{ Adult } \\
\cline { 2 - 3 } & Non-social & & Pro-social & & Non-social \\
\hline Mean Imitation Effect (SEM) & $50.62(11.99)$ & $37.98(9.23)$ & & $37.98(7.19)$ & $71.11(11.79)$ \\
Mean Effector Priming Effect (SEM) & $38.55(8.02)$ & $35.23(9.25)$ & & $49.44(8.28)$ & $55.55(14.84)$ \\
\hline
\end{tabular}

The Imitation Effect is the RT difference between incompatible (e.g. Participant lifts index finger and watches a video of a middle finger lift) and compatible (e.g. Participant lifts index finger and watches an index finger lift) trials in the Imitation Task. The Effector Priming Effect is the RT difference between incompatible (e.g. Participant lifts index finger and observes a middle finger changing colour) and compatible (e.g. Participant lifts index finger and observes an index finger changing colour) trials in the Effector Priming Control task 
$t$-tests revealed that for the Adult Group the Imitation Effect was significantly greater for the Pro-social Group (Mean = 71.11, SEM = 11.79) than for the Non-social Group $($ Mean $=37.98$, SEM $=7.19 ; t(54)=-2.40, P<0.01$, 1-tailed). No such significant difference between Prime Groups existed for the Adolescent Age Group (Fig. 2: Pro-social: $\quad$ Mean $=37.98, \quad \mathrm{SEM}=9.23$; Non-social: Mean $=50.62, \quad$ SEM $=11.99 ; t(32)=0.84, P=0.41)$. Hence, social priming differentially affected imitation in adolescents and adults.

Specificity of social priming effects on mechanisms of imitation

Paired samples $t$ tests on compatible and incompatible RTs for Effector Priming Control trials demonstrated that each of the four groups exhibited a significant Effector Priming Effect (Adolescent Non-social Group incompatible trials mean RT $(\mathrm{SEM})=570.91$ (21.42), compatible trials mean $\mathrm{RT}(\mathrm{SEM})=532.36(19.73), \quad t(16)=4.81, \quad P<0.001$, 1-tailed; Adolescent Pro-social Group incomp mean RT $(\mathrm{SEM})=540.04$ (17.81), comp mean RT $(\mathrm{SEM})=504.82$ (19.76), $t(16)=3.81, P<0.01$, 1-tailed; Adult Non-social Group incomp mean RT $(\mathrm{SEM})=532.22$ (15.61), comp mean RT $(\mathrm{SEM})=482.77(13.44), t(27)=5.97, \quad P<$ 0.001, 1-tailed; Adult Pro-social Group incomp mean RT $(\mathrm{SEM})=571.12(29.59)$, comp mean $\mathrm{RT}(\mathrm{SEM})=515.57$ (20.91), $t(27)=3.74, P<0.001,1$-tailed). That is, for each group, responses were faster when the same finger changed colour than when a different finger changed colour.

Data from the Imitation task show that adult participants are significantly affected by social priming, but that adolescent participants are not. In order to confirm that the social priming effect on imitation seen in adults is specific

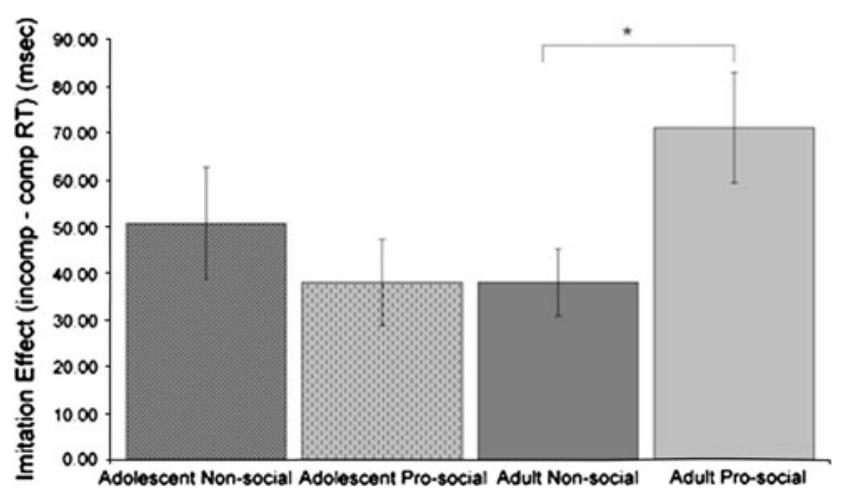

Fig. 2 Imitation Effects for Adolescent and Adult Pro- and Nonsocial Prime Groups. The ANOVA revealed a significant interaction between Age and Prime Group. Post hoc $t$ tests revealed that for the Adult Group the Imitation Effect was significantly greater for the Prosocial Group than for the Non-social Group. No such significant difference between Prime Groups existed for the Adolescent Age Group to imitation (as found by Leighton et al. 2010), we conducted a $2 \times 2$ ANOVA with between-subjects factor Prime Group (Pro-social, Non-social) and within-subjects factor Experimental Task (Imitation task, Effector Priming Control task) for the Adult Group. There was a significant interaction between Prime Group and Experimental Task $(F(1,54)=2.89, P<0.05,1$-tailed $)$. The same analysis conducted on the Adolescent Pro- and Non-social Groups (which do not differ in terms of imitation) did not show a significant interaction $(F(1,32)=0.30, P=0.58)$. $T$-tests showed that for the Adult Group the interaction was driven by a significant difference in the magnitude of the Imitation Effect for the Pro-social $($ Mean $=71.11$, SEM $=11.79$ ) compared to Non-social Group (Mean = 37.98, SEM = $7.19 ; t(54)=-2.40, P<0.01,1$-tailed) but no significant difference between the Prime Groups in terms of the Effector Priming Effect (Fig. 3: Pro-social: Mean $=55.55$, $\mathrm{SEM}=14.84$; Non-social: Mean $=49.44, \mathrm{SEM}=8.28$; $t(54)=-0.36, \quad P=0.36)$. The three-way interaction between Prime Group (Pro-social, Non-social), Age Group (Adolescent, Adult) and Experimental Task (Imitation task, Effector Priming Control task) approached significance $(F(F(1,86)=2.22, \quad P=0.07, \quad 1$-tailed $)$. The preceding analyses were repeated on the raw RT data, including a repeated measures factor of Compatibility (Compatible, Incompatible) in order to assess whether Prime Group, Age Group, or the interaction between these factors affected RT irrespective of Compatibility. These effects were all nonsignificant (all $P$ s $>0.1$ ).

In order to assess the mechanism by which social priming affects imitation, the relationship between response speed and the social priming effect in each age group was examined. Reaction times were sorted according to the factors of Compatibility, Age Group and Prime Group and independently separated into three time bins, representing fast, medium and slow responses. The

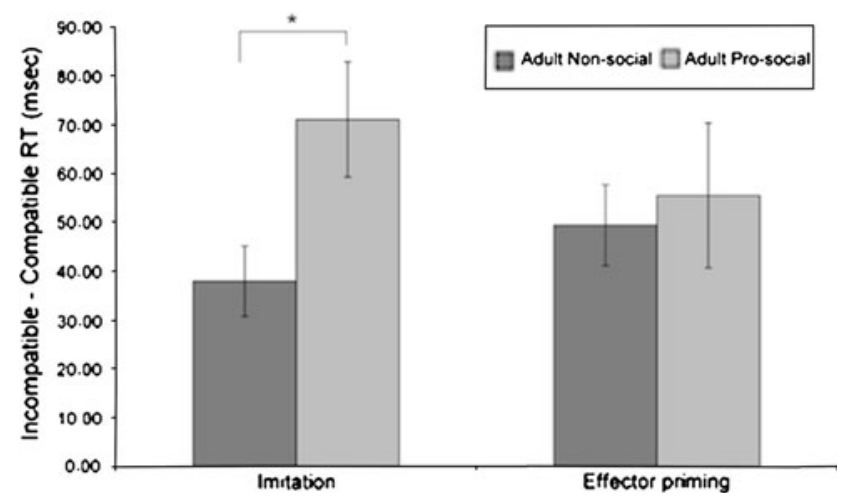

Fig. 3 For the Adult Group, there was a significant difference in the magnitude of the Imitation Effect for the Pro-social compared to Nonsocial Prime Group but no significant difference between the Prime Groups in terms of the Effector Priming Effect 
difference between mean Compatible and Incompatible reaction times at each time bin was entered into a $3 \times 2 \times 2$ ANOVA with factors of Speed (Fast, Medium, Slow), Age Group (Adult, Adolescent) and Prime Group (Pro-social, Non-social). As Mauchley's test of sphericity was significant, degrees of freedom were corrected using the Greenhouse-Geisser method. Results of this ANOVA revealed a significant Speed x Age Group x Prime Group Interaction $(F(1.2,97.7)=6.0, P=0.01)$. Inspection of the means (Fig. 4) of each condition at each of the time bins suggests that the effect of pro-social priming is seen only in the Adult Group at the slowest reaction times. The interaction between Speed, Age and Prime Group was decomposed by performing ANOVAs with factors of Age and Prime Group at each of the 3 time bins. There was a significant interaction between Age and Prime Group at the slowest time bin $(F(1,83)=6.0, P=0.01)$, but not at the medium $(F(1,83)=2.7, P=0.10)$, or fast time bins $(F(1,83)<1)$, although the interaction approached significance at the medium time bin. These analyses were followed-up with univariate ANOVAs with a between-groups factor of Prime Group at each combination of the Speed and Age Group factors. The effect of Prime Group was not significant for any time bin in the Adolescent Group (all $P$ s $>0.244)$. The effect of Prime Group was not significant in the Adult Group at the fastest time bin $(F(1,54)=1.7$, $P=0.2$ ), approached significance at the medium time bin, $(F(1,54)=2.9, P=0.09)$, and was significant at the slowest time bin $(F(1,54)=6.4, P=0.01)$.

\section{Discussion}

Previous research has demonstrated that in adults priming a positive social attitude in comparison with a negative social

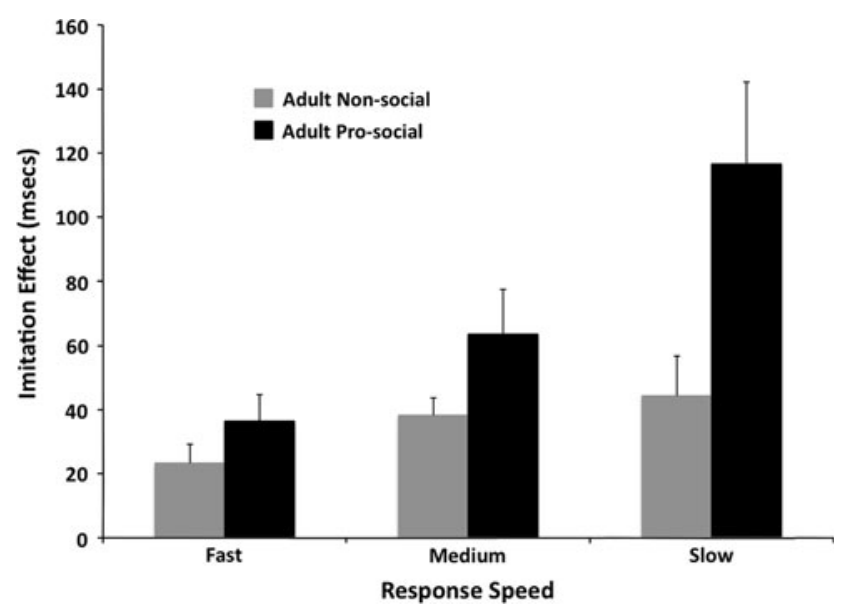

Fig. 4 Social priming affects slow, but not medium or fast, responses in adult participants. This suggests that social priming affects the inhibition of imitative responses attitude promotes imitation (Leighton et al. 2010). Since social attitudes and the social brain are changing throughout the adolescent years, the aim of this study was to test whether pro-social attitudes promote imitation in an Adolescent Group to the same extent as in an Adult Group. In agreement with the results of Leighton et al., we found that adults who had been primed with words promoting pro-social attitudes (e.g. friend, crowded, team, talkative) showed a greater Imitation Effect than adults who had been primed with words promoting non-social attitudes (e.g. himself, solo, one, private). The difference in the degree of modulation of imitation by social priming was significantly greater for adults than for adolescents. Indeed, there was no significant difference between the amount of imitation shown by adolescents primed with pro-social words compared with adolescents primed with non-social words.

It is unlikely that the inefficacy of pro-social priming on imitation for the Adolescent Group is due to an ineffective pro-social priming technique. The prime words in the Priming task were selected on the basis that they feature in the common vocabulary of both adolescent and adult participants. Throughout the testing sessions, none of the participants questioned the definition of the words. Furthermore, statistical analyses demonstrated that for all groups the average error rate on the Priming task did not exceed 2 of 32 sentences, and there was no significant difference between the error rates generated by the four groups. Hence, all groups were able to use the scrambled words to create a grammatically correct sentence.

The associative sequence learning model (Heyes 2001, 2003; Heyes and Ray 2000; Brass and Heyes 2005) suggests that imitation is mediated by low-level mechanisms in which activation of a sensory representation of a body movement (e.g. by visual attention to that movement) necessarily results in activation of a motor representation of the same movement (and thereby an impulse to perform the movement), provided that the sensory and motor representations have become linked through previous, correlated experience of observing and executing the movement. In principle, social attitudes could modulate imitation via an attentional route-by influencing the probability that sensory representations of body movement will be activated (see also Teufel et al. 2010)_or via an inhibitory route-by influencing the degree to which activation of motor representations by associated sensory representations is suppressed to prevent overt performance of the represented movement (see also Liepelt and Brass 2010). Leighton et al. (2010) argue that positive social attitudes most likely promote imitation via an inhibitory route rather than an attentional route. They argue that the attentional route is less likely since imitation tasks such as the one used in the current experiment require attention to the same part of the screen as the movement stimuli (i.e. in the 
current experiment the cue to move was presented equidistant from the index and middle fingers of the video hand); also any trials in which participants did not attend are detected by checking for incorrect responses and for abnormally long or short reaction times, and these trials are excluded from the analysis.

The suggestion that social priming affects inhibition of imitative responses was investigated in the current data by performing a 'bin analysis' on the reaction time data from the Imitation Task to investigate whether response speed interacts with the effect of the priming manipulation. The results of the bin analysis revealed that the effect of social priming, that was evident in adults, was seen only for slower responses. Following previous studies (see Catmur and Heyes 2010), we suggest that this pattern of data fits a model of social priming affecting late processes related to inhibition, rather than an attentional process (where effects of social priming may be expected to be seen for even the fastest responses). Our results therefore suggest that, for adults, positive social attitude priming leads to the release of inhibition of imitative motor output and that this process does not occur for adolescents. At present, the functional mechanism by which imitation inhibition is reduced by pro-social priming is unknown; however, Brass et al. (2005) argue that imitation inhibition relies on appropriate distinction between motor programmes initiated by the self, and those initiated by observation of the other. It is possible therefore that the effect of pro-social priming is to blur the distinction between the self and other and thus reduce the potential for imitation inhibition.

Previous research has shown that being imitated promotes pro-social behaviour (Chartrand and Bargh 1999), and consequently, it has been suggested that imitation is a non-conscious tool that individuals use instinctively to facilitate social interactions with others (Cheng and Chartrand 2003; Lakin and Chartrand 2003; Chartrand and Jeffers 2003). Kühn et al. (2010) investigated the mechanism by which being imitated may lead to pro-social behaviour using fMRI and found that observing another individual being imitated activated neural structures involved in reward processing such as the ventral MPFC and medial orbitofrontal cortex. These areas are some of the latest to develop and are structurally and functionally immature in adolescence (e.g. Blakemore et al. 2007; Shaw et al. 2008). Leighton et al. (2010) have suggested that imitation and social attitudes are part of a positive feedback loop in which pro-social attitudes generate imitation, and imitation and/or being imitated further increases positive social attitudes. Leighton et al. propose that this relationship may be either innate or a product of social learning: humans may have an innate tendency to mimic more when they are in an affiliative frame of mind, or they may learn through interaction with others that it is under these circumstances that imitation tends to be most rewarding. Accordingly, it may be the case that the adolescent brain develops in accordance with genetically pre-programmed changes and that the neural machinery for associating positive social attitudes with imitation is not fully developed until adulthood. Alternatively, it may be the case that as adolescents gain more experience with the imitation/ positive social attitude positive feedback loop described by Leighton et al. the association between imitation and positive social attitudes is strengthened. Studies showing that learning is often accompanied by neural changes (see Galvan 2010 for recent review) highlight the possibility that the strengthening of an association between imitation and positive social attitudes may play a part in the development of the adolescent brain. Further studies are necessary to gauge relative contributions of genetically preprogrammed developmental changes and those triggered by changes in the social environment.

The original, naturalistic, experiments demonstrating a link between pro-social attitudes and imitation (e.g. Lakin and Chartrand 2003) could not dissociate whether the effect of pro-social priming was specific to imitation or whether it could be explained by effector priming. Leighton et al. suggest that the effect is specific to imitation since pro-socially primed adults were faster to conduct a handopen response when observing a hand-open response rather than being faster to open their hand when observing any hand movement. However, the paradigm used by Leighton et al. did not include a test of effector priming and therefore cannot show whether pro-social priming acts specifically on imitation. To directly test whether social attitudes modulate effector priming, we included an Effector Priming Control task. Supporting the hypothesis that social priming would have a specific effect on imitation, our Adult Group demonstrated a modulation of imitation by social priming, whereas effector priming was unaffected. The specific effects of social attitude priming on imitation are in line with the theory that imitation inhibition is a separate ability, unrelated to performance on more classical inhibition tasks (Brass et al. 2003) and which results in activation of distinct neural areas (Brass et al. 2005).

\section{Conclusion}

The current study shows that whereas pro-socially primed adults show greater imitation than non-socially primed adults, this effect was not found for an Adolescent Group. The lack of the social priming effect for the Adolescent Group may be a result of the immature development of social brain regions and/or of reduced experience of imitating and being imitated and the positive social attitudes that this can generate. Analysis of our Effector Priming 
Control task demonstrates that the effect of social priming that can be seen in the Adult data is specific to the mechanisms of imitation.

Acknowledgments JC was funded by the Wellcome Trust FourYear PhD Programme in Neuroscience at UCL. The authors declare that they have no conflict of interest. The authors would like to thank Sarah-Jayne Blakemore, Clare Press and two anonymous reviewers for their helpful comments on earlier drafts of the manuscript.

Open Access This article is distributed under the terms of the Creative Commons Attribution Noncommercial License which permits any noncommercial use, distribution, and reproduction in any medium, provided the original author(s) and source are credited.

\section{References}

Bach P, Tipper SP (2007) Implicit action encoding influences personal-trait judgments. Cognition 102:151-178

Bailenson JN, Yee N (2005) Digital chameleons: automatic assimilation of nonverbal gestures in immersive virtual environments. Psychol Sci 16(10):814-819

Berger SM, Hadley SW (1975) Some effects of a model's performance on an observer's electromyographic activity. Am J of Psychol 88:263-276

Bernieri FJ (1988) Coordinated movement and rapport in teacherstudent interactions. J Nonverbal Behav 12:120-138

Blakemore SJ (2008) The social brain in adolescence. Nat Rev Neurosci 9(4):267-277

Blakemore SJ (2010) The developing social brain: implications for education. Neuron 65(6):744-747

Blakemore S, den Ouden H, Choudhury S, Frith C (2007) Adolescent development of the neural circuitry for thinking about intentions. Soc Cog Affect Neurosci 2(2):130-139

Brass M, Heyes CM (2005) Imitation: is cognitive neuroscience solving the correspondence problem? Trends Cogn Sci 9:489 495

Brass M, Bekkering H, Wohlschlager A, Prinz W (2000) Compatibility between observed and executed finger movements: comparing symbolic, spatial, and imitative cues. Brain Cogn 44:124-143

Brass M, Derrfuss J, Matthes-von Cramon G, von Cramon DY (2003) Imitative response tendencies in patients with frontal brain lesions. Neuropsychology 17(2):265-271

Brass M, Derrfuss J, von Cramon DY (2005) The inhibition of imitative and overlearned responses: a functional double dissociation. Neuropsychologia 43:89-98

Brothers L (1990) The social brain: a project for integrating primate behavior and neurophysiology in a new domain. Concepts in Neurosci 1:27-51

Brown BB (2004) Adolescents' relationships with peers. In: Lerner RM, Steinberg L (eds) Handbook of adolescent psychology, 2nd edn. Wiley, Hoboken, NJ

Burnett S, Bird G, Moll J, Frith C, Blakemore S (2009) Development during adolescence of the neural processing of social emotion. J Cogn Neurosci 21(9):1736-1750

Catmur C, Heyes CM (2010) Time course analyses confirm independence of imitative and spatial compatibility. J Exp Psychol Hum Percept Perform. doi:10.1037/a0019325

Chartrand TL, Bargh JA (1999) The chameleon effect: the perceptionbehavior link and social interaction. J Pers Soc Psychol 76(6):893-910
Chartrand TL, Jeffers V (2003) Consequences of automatic goal pursuit and the case of nonconscious mimicry. In: Forgas JP, Williams KD, von Hippel W (eds) Responding to the social world: implicit and explicit processes in social judgments and decisions. Psychology Press, Philadelphia

Cheng CM, Chartrand TL (2003) Self-monitoring without awareness: using mimicry as a nonconscious affiliation strategy. J Pers Soc Psychol 85(6):1170-1179

Frith CD (2007) The social brain? Philos Trans R Soc Lond B Biol Sci 362(1480):671-678

Frith U, Frith C (2010) The social brain: allowing humans to boldly go where no other species has been. Philos Trans R Soc Lond B Biol Sci 365(1537):165-176

Galvan A (2010) Neural plasticity of development and learning. Hum Brain Mapp 31(6):879-890

Giedd JN, Blumenthal J, Jeffries NO, Castellanos FX, Liu H, Zijdenbos A, Paus T, Evans AC, Rapoport JL (1999) Brain development during childhood and adolescence: a longitudinal mri study. Nat Neurosci 2(10):861-863

Gillmeister H, Catmur C, Liepelt R, Brass M, Heyes CM (2008) Experience-based priming of body parts: a study of action imitation. Brain Res 1217:157-170

Harter S (1990) Developmental differences in the nature of selfrepresentations: implications for the understanding, assessment, and treatment of maladaptive behaviour. Cogn Therapy Res 14(2):113-142

Heyes CM (2001) Causes and consequences of imitation. Trends Cogn Sci 5:253-261

Heyes CM (2003) Four routes of cognitive evolution. Psychol Rev 110:713-727

Heyes CM, Ray ED (2000) What is the significance of imitation in animals? Advances in the Study of Behavior 29:215-245

Heyes CM, Bird G, Johnson H, Haggard P (2005) Experience modulates automatic imitation. Brain Res Cogn Brain Res 22(2):233-240

Hillebrandt H, Sebastian C, Blakemore S (in press) Experimentally induced social inclusion influences behavior on trust games. Cogn Neurosci. doi:10.1080/17588928.2010.515020

Huttenlocher PR (1979) Synaptic density in human frontal cortexdevelopmental changes and effects of aging. Brain Res 163(2):195-205

Kühn S, Müller BCN, van Baaren RB, Wietzker A, Dijksterhuis A, Brass M (2010) Why do I like you when you behave like me? Neural mechanisms mediating positive consequences of observing someone being imitated. Soc Neurosci 5(4):384-392

Lafrance M (1979) Nonverbal synchrony and rapport: analysis by the cross-lag panel technique. Soc Psychol Quart 42:66-70

LaFrance M (1982) Posture mirroring and rapport. In: Davis M (ed) Interaction rhythms: periodicity in communicative behaviour. Human Sciences Press, New York

Lafrance M, Broadbent M (1976) Group rapport: posture sharing as a nonverbal indicator. Group Organization Studies 1:328-333

Lakin JL, Chartrand TL (2003) Using nonconscious behavioral mimicry to create affiliation and rapport. Psychol Sci 14(4):334 339

Leighton J, Bird G, Orsini C, Heyes CM (2010) Social attitudes modulate automatic imitation. J Exp Soc Psychol. doi:10.1016/ j.jesp.2010.07.001

Liepelt R, Brass M (2010) Top-down modulation of motor priming by belief about animacy. Exp Psychol 57(3):221-227

Paus T (2005) Mapping brain maturation and cognitive development during adolescence. Trends Cogn Sci 9(2):60-68

Sebanz N, Knoblich G, Prinz W (2003) Representing others' actions: just like one's own? Cognition 88(3):B11-B21

Sebanz N, Bekkering H, Knoblich G (2006) Joint action: bodies and minds moving together. Trends Cogn Sci 10(2):70-76 
Sebastian C, Burnett S, Blakemore SJ (2008) Development of the self-concept during adolescence. Trends Cogn Sci 12(11): $441-446$

Sebastian C, Viding E, Williams KD, Blakemore S (2010) Social brain development and the affective consequences of ostracism in adolescence. Brain Cog 72(1):134-145

Shaw P, Kabani NJ, Lerch JP, Eckstrand K, Lenroot R, Gogtay N, Greenstein D, Clasen L, Evans A, Rapoport JL, Giedd JN, Wise SP (2008) Neurodevelopmental trajectories of the human cerebral cortex. J Neurosci 28(14):3586-3594

Sowell ER, Thompson PM, Holmes CJ, Batth R, Jernigan TL, Toga AW (1999) Localizing age-related changes in brain structure between childhood and adolescence using statistical parametric mapping. Neuroimage 9(6 Pt 1):587-597

Spengler S, von Cramon DY, Brass M (2010a) Resisting motor mimicry: control of imitation involves processes central to social cognition in patients with frontal and temporo-parietal lesions. Soc Neurosci 5(4):401-416
Spengler S, Bird G, Brass M (2010b) Hyper-imitation of actions is related to reduced understanding of others' minds in autism spectrum conditions. Biol Psychiatry 68(12):1148-1155

Teufel C, Fletcher PC, Davis G (2010) Seeing other minds: attributed mental states influence perception. Trends Cogn Sci 14(8):376382

van Baaren RB, Holland RW, Steenaert B, van Knippenberg A (2003a) Mimicry for money: behavioral consequences of imitation. J Exp Soc Psych 39(4):393-398

van Baaren RB, Maddux WW, Chartrand TL, de Bouter C, van Knippenberg A (2003b) It takes two to mimic: behavioral consequences of self-construals. J Pers Soc Psychol 84(5): 1093-1102

van Baaren RB, Holland RW, Kawakami K, van Knippenberg A (2004) Mimicry and prosocial behavior. Psychol Sci 15(1):71-74

Wang AT, Lee SS, Sigman M, Dapretto M (2006) Developmental changes in the neural basis of interpreting communicative intent. Soc Cog Affect Neurosci 1(2):107-121 